\title{
Die Rechte der Antragsteller in den Förderverfahren der DFG
}

\begin{abstract}
Die zunehmende Honorierung von Drittmitteln hat die Bedeutung der DFG erheblich gestärkt. Gleichzeitig werden Antragsteller in DFG-Förderverfahren weitgehend als rechtlos behandelt. Ob diese Vorgehensweise, die vor allem im Hinblick auf die Wissenschaftsfreiheit Bedenken hervorruft, haltbar ist, wird im Rahmen des Aufsatzes untersucht. Außerdem wird erörtert, ob die Gewährleistungspflicht des Art. 5 Abs. 3 GG den Staat verpflichtet, auf eine Verbesserung der Rechtsposition der Antragsteller in den DFG-Förderverfahren hinzuwirken.
\end{abstract}

\section{Einleitung}

Die Umstellung der Hochschulfinanzierung auf eine stärker leistungsabhängige, outputorientierte Ressourcenverteilung ist ein wesentliches Element der Hochschulreformen seit Ende der 90er Jahre. Deutlich sichtbar wird dieser Paradigmenwechsel vor allem daran, dass die staatlichen Zuweisungen an die Hochschulen verstärkt anhand von Indikatoren vergeben werden. So werden beispielsweise in Nordrhein-Westfalen $20 \%$ des staatlichen Globalzuschusses an die Hochschulen nach den Indikatoren Absolventen (50\%), Promotionen (10\%) und Drittmittel (40\%) verteilt. Andere Bundesländer haben ähnliche Modelle eingeführt. ${ }^{1}$ Auch innerhalb der Hochschulen erfolgt die Mittelvergabe stärker leistungsbezogen. Bereits 2005 setzten nach einer Befragung der HIS knapp $90 \%$ der Universitäten Formelmodelle für die Mittelzuweisungen an die Fakultäten ein. ${ }^{2}$ Der Anteil der so vergebenen Gelder ist indes derzeit noch überschaubar. Er liegt nur in Ausnahmefällen über $10 \%$ der staatlichen Zuschüsse. ${ }^{3}$ Innerhalb der Hochschulen liegt die durchschnittliche Gewichtung der Forschungsindikatoren bei $38 \%{ }^{4} \mathrm{Ge}$ nutzt werden vor allem Drittmittel (100\%) und Promotionen bzw. Habilitationen (80\%). Nur selten wird die Zahl der Publikationen (16\%) verwendet. ${ }^{5}$ Somit belohnen sowohl die staatlichen als auch die universitären Vergabemodelle im Forschungsbereich vor allem Erfolge in der Drittmitteleinwerbung. Die These, dass Karrieren in der Wissenschaft und die Zukunft ganzer Institute mehr und mehr vom Umfang der eingeworbenen Drittmittel abhängen, ${ }^{6}$ ist infolgedessen naheliegend.

Für die Gewährleistung der Wissenschaftsfreiheit ist daher insbesondere die Pluralität der Drittmittelgeber wichtig. Nach der Untersuchung des Wissenschaftsrates aus dem Jahr 2000 werden jeweils $25 \%$ der Drittmittel von Einrichtungen des Bundes und aus

1 Jaeger, Zeitschrift für Hochschulentwicklung 2008, 89 (90 f.).

2 Leszczensky/Jaeger/Orr/Schwarzenberger, Formelgebundene Mittelvergabe und Zielvereinbarungen als Instrumente der Budgetierung an deutschen Universitäten: Ergebnisse einer bundesweiten Befragung, 2005, S. 12.

3 Vgl. Leszczensky/Jaeger/Orr/Schwarzenberger (o. Fußn. 2), S. 14 f.

4 Leszczensky/Jaeger/Orr/Schwarzenberger (o. Fußn. 2), S. 20 f.

5 Leszczensky/Jaeger/Orr/Schwarzenberger (o. Fußn. 2), S. 20 f.

6 www.spiegel.de/wissenschaft/mensch/0,1518,394373,00.html (Stand: 11.8.2009). 
dem Bereich der Wirtschaft vergeben. Stiftungen und Fördergesellschaften spielen mit knapp $5 \%$ der vergebenen Drittmittel lediglich eine untergeordnete Rolle. Schon bei einer bereichsspezifischen Betrachtungsweise ist die DFG mit 35\% der wichtigste Drittmittelgeber. ${ }^{7}$ Im Wettbewerbsrecht begründet ein solcher Marktanteil regelmäßig eine marktbeherrschende Stellung ( $\$ 19$ Abs. 3 GWB). Aufgrund der damit verbundenen Einschränkung der Pluralität der Drittmittelgeber ist es schwierig, diese gegeneinander auszuspielen, um so die Abhängigkeit von einzelnen Drittmittelgebern zu verringern. ${ }^{8}$ Für die Gewährleistung der Wissenschaftsfreiheit ist deshalb die Ausgestaltung der Vergabeverfahren der DFG von essentieller Bedeutung. Bedenklich ist daher, dass Antragstellern gegenüber der DFG bislang praktisch keine Ansprüche gewährt werden. Deutlich wurde dies zuletzt im Fall des Siegener Chemieprofessors Harro Lentz, der die DFG erfolglos auf Herausgabe der Begutachtungen seines Antrags verklagt hatte. ${ }^{9}$ Das Amtsgericht Bonn begründete dies damit, dass die DFG als ein eingetragener Verein und damit als juristische Person des Privatrechts keinen öffentlich-rechtlichen Bindungen unterliege. ${ }^{10}$

$\mathrm{Ob}$ diese Ansicht haltbar ist, wird nachfolgend untersucht. Dazu werden zunächst die Förderprogramme und Vergabeverfahren der DFG erläutert (II.). Entscheidend für die Rechtsstellung der Antragsteller ist die Einordnung dieser Vergabeverfahren in die Rechtsgebietsdogmatik (III.) Dafür werden die unterschiedlichen möglichen Beteiligungsformen dargestellt, bevor die konkrete Zuordnung zum öffentlichen oder zum Privatrecht erfolgt. Im Anschluss werden einzelne zentrale Rechte der Antragsteller näher untersucht (IV.). Abschließend wird der Frage nachgegangen, ob mit der derzeitigen Ausgestaltung der Vergabeverfahren durch die DFG der Staat seine Schutzpflicht aus Art. 5 Abs. 3 GG verletzt hat (V.).

\section{Aufbau der Vergabeverfahren der DFG}

Schwerpunkte der Fördertätigkeit der DFG stellen die Einzelförderung und die Unterstützung von koordinierten Programmen dar. Für die Einzelförderung wurden im Jahr 2008810 Mio. Euro verausgabt. Als koordinierte Programme wurden insbesondere Sonderforschungsbereiche mit 540 Mio. Euro und die Projekte der Exzellenzinitiative mit 342 Mio. Euro unterstützt. ${ }^{11}$ Anträge im Normalverfahren können alle promovierten Wissenschaftler an deutschen Forschungseinrichtungen stellen. Nach Antragseingang wählt der jeweils zuständige Programmdirektor der DFG-Geschäftsstelle vornehmlich nach dem Kriterium der Expertise zum Gegenstand des Antrags zwei Gutachter aus, die ein schriftliches Votum abgeben. Sowohl die Voten als auch die Namen der Begutachtenden bleiben gegenüber dem Antragsteller geheim. Die Begutachtungen werden den Fachkollegien ${ }^{12}$ vorgelegt, die eine vergleichende Bewertung aller Anträge in einem

7 Wissenschaftsrat, Drittmittel und Grundmittel der Hochschulen 1993 bis 1998, Drs. 4717/00, 2000, S. 59ff.

8 Stichweh, in: Sieg/Korsch, Die Idee der Universität heute, 2005, S. 123 (130 f.).

9 www.spiegel.de/wissenschaft/mensch/0,1518,394373,00.html (Stand: 11.8.2009).

10 AG Bonn, Urt. v. 21.12.2005 - 9 C 390/05.

11 DFG-Jahresbericht 2008, S. 198ff.

12 Ausführlich zu den Fachkollegien Koch, WissR 2006, 25 (37ff.). 
Fach vornehmen. Sie geben eine Förderempfehlung ab, über die der Hauptausschuss der DFG abschließend entscheidet. Die Förderquote liegt derzeit bei knapp 53 Prozent. ${ }^{13}$ Der Antragsteller wird über das Ergebnis durch die DFG-Geschäftsstelle informiert.

Im Gegensatz dazu stellen die Vergabeverfahren für die koordinierten Programme größere Anforderungen an die Antragsteller. Auch sind sie aufwendiger gestaltet. Antragsberechtigung für die Förderung von Sonderforschungsbereichen besitzen z. B. nicht einzelne Wissenschaftler, sondern ausschließlich Hochschulen. Im Gegensatz zum Normalverfahren handelt es sich außerdem nicht um ein rein schriftliches Verfahren. Vielmehr findet nach Einreichung des Antrags eine Begutachtung am Ort der antragstellenden Hochschule statt. Auf Grundlage der Begutachtungsergebnisse trifft im Anschluss der Bewilligungsausschuss für die Sonderforschungsbereiche, welcher sich aus Wissenschaftlern und Vertretern der zuständigen Bundes- und Landesministerien zusammensetzt, die Förderentscheidung. Ähnlich ist das Vergabeverfahren der Exzellenzinitiative aufgebaut, welches die DFG nach § 4 Abs. 1 der Bund-Länder-Vereinbarung über die Exzellenzinitiative (ExV) durchführt. Allerdings erfolgt die Entscheidung über die Förderung von Graduiertenschulen, Exzellenzclustern und Zukunftskonzepten in zwei Stufen. Zuerst wird eine Antragsskizze eingereicht. Die Antragsskizzen für Graduiertenschulen und Exzellenzcluster werden durch externe Gutachter geprüft. Dagegen wird die Begutachtung der Zukunftskonzepte von der Strategiekommission, welche aus Vertretern des Wissenschaftsrates besteht, durchgeführt. Die endgültige Auswahl der Skizzen trifft die aus Vertretern der DFG und des Wissenschaftsrates zusammengesetzte Gemeinsame Kommission (§ 4 Abs. 6 S. 2 ExV). Die Vollanträge werden erneut durch Externe begutachtet. Auf dieser Grundlage gibt die Gemeinsame Kommission gemäß $§ 4$ Abs. 7 S. 1 ExV eine Förderempfehlung ab. Die endgültige Entscheidung obliegt nach $\S 4$ Abs. 8 S. 1 BLV dem Bewilligungsausschuss, dem neben den Mitgliedern der Gemeinsamen Kommission auch staatliche Vertreter angehören. Die ab 2011 geltende Neuauflage der Exzellenzinitiative, die am 4.4.2009 beschlossen wurde, sieht ein fast identisches Vergabeverfahren vor.

\section{Die Förderverfahren der DFG aus rechtsgebietsdogmatischer Sicht}

Wie die Förderentscheidungen der DFG in die Rechtsgebietsdogmatik, also öffentlich oder privatrechtlich, einzuordnen sind, ist essentiell für den Umfang der rechtlichen Anforderungen an diese. Als eingetragener Verein kann die DFG prinzipiell gegenüber Wissenschaftlern und Hochschulen als private Institution zur Erfüllung eigener, privater Aufgaben fungieren. Angesichts des Umstandes, dass die DFG mit der Wissenschaftsförderung eine öffentliche Aufgabe wahrnimmt und die dazu aufgewandten Gelder fast ausschließlich von Bund und Ländern stammen, bestehen trotzdem Zweifel, ob die Vergabeentscheidungen als rein privatrechtlich einzustufen sind. Zunächst werden daher die möglichen Beteiligungsformen Privater in staatlich finanzierten Förderverfahren und die Kriterien zu ihrer Abgrenzung entwickelt, bevor die Vergabeentscheidungen der DFG in diese eingeordnet werden.

13 DFG-Jahresbericht 2008, S. 154. 


\section{Beteilungsformen Privater in staatlich finanzierten Förderungsverfahren}

Die Einschaltung Privater zur Vorbereitung und Abwicklung staatlicher Förderprogramme ist im Wissenschaftsbereich keine Seltenheit. So organisiert z. B. das Deutsche Elektronen-Synchrotron (DESY) die Projektförderung des BMBF im Bereich der naturwissenschaftlichen Grundlagenforschung. ${ }^{14}$ Der Grund für die Einschaltung ist vor allem der unzureichende Sachverstand für die Bewertung hochspezialisierter Forschungsprogramme in den Ministerien. ${ }^{15}$ Bei der Art der Beteiligung Privater besitzen die Ministerien in gewissem Umfang Gestaltungsfreiheit. ${ }^{16}$ Sie können diese entweder als Projektträger integrieren oder zu Zuwendungsempfängern machen.

Projektträger nehmen bei der Projektbetreuung keine eigenen Aufgaben, sondern solche des Staates wahr. ${ }^{17}$ Es gibt zwei Grundtypen von Projektträgern. So können die Privaten als unselbständiger Verwaltungshelfer fungieren. Diese können zwar im Vorfeld der Vergabeentscheidung und bei deren Ausführung tätig werden, sind aber nicht befugt, selbst im eigenen Namen einen Zuwendungsbescheid zu erlassen. ${ }^{18}$ Eine Rechtsbeziehung zwischen dem Verwaltungshelfer und dem Zuwendungsempfänger entsteht nicht. ${ }^{19}$ Anderes gilt für beliehene Projektträger. Sie sind Behörden im Sinne von $\S 1$ Abs. 4 VwVfG, weshalb bei der Vergabeentscheidung ein öffentlich-rechtliches Rechtsverhältnis zwischen dem Projektträger und dem Zuwendungsempfänger entsteht. ${ }^{20}$

Neben der Beteiligung als Projektträger können die Privaten selbst zum Zuwendungsempfänger werden (sog. Mittlerorganisationen). ${ }^{21}$ Solche Organisationen nehmen eine „Zwitterrolle“ ein. Auf der einen Seite arbeiten sie mit öffentlichen Geldern und haben häufig staatliche Vertreter in ihren Gremien, wodurch sie einem finanziellen und personellen Staatseinfluss unterliegen. Auf der anderen Seite werden die konkreten Förderentscheidungen in der Regel auf Grundlage wissenschaftlicher Begutachtungen durch vornehmlich nach wissenschaftlichen Kriterien zusammengesetzte Gremien getroffen. ${ }^{22}$ Vorteile der Einschaltung von Mittlerorganisationen sind, dass die Eigengesetzlichkeiten der Wissenschaft besser berücksichtigt werden können und das staatliche Neutralitätsgebot leichter gewahrt werden kann. ${ }^{23}$ Die Weiterleitung der Gelder durch die Mittlerorganisationen an die Endempfänger kann sowohl in privatrechtlicher als auch in öffentlich-rechtlicher Form erfolgen. Letzteres erfordert ebenfalls eine Beleihung. Inwieweit dies bei wissenschaftlichen Mittlerorganisationen zulässig ist, ist umstritten. ${ }^{24}$ Indes ist diese Frage nicht von praktischer Relevanz, da ein Beleihungsakt der

14 Vgl. http://pt.desy.de/ (Stand: 11.8.2009).

15 Dommach, in: Heuer, Haushaltsrecht, Loseblatt 2008, § 44 BHO Rn. 78; Dittrich/Lamm, in: Köckritz/Ermisch, Bundeshaushaltsordnung, Loseblatt 2007, § 44 BHO Tz. 79.

16 Dittrich/Lamm (o. Fußn. 15), §44 BHO Tz. 79.13.

17 Dittrich/Lamm (o. Fußn. 15), § 44 BHO Tz. 79.

18 Dittrich/Lamm (o. Fußn. 15), § 44 BHO Tz. 79.1; Dommach (o. Fußn. 15), § 44 BHO Rn. 78.

19 Streiter, Wissenschaftsförderung durch Mittlerorganisationen, 2008, S. 239.

20 Dittrich/Lamm (o. Fußn. 15), §44 BHO Tz. 79.2.

21 Dittrich/Lamm (o. Fußn. 15), § 44 BHO Tz. 79.13.

22 Streiter (o. Fußn. 19), S. 5 f.

23 Streiter (o. Fußn. 19), S. 255.

24 Bejahend: Streiter (o. Fußn. 19), S. 211; ablehnend: Meusel, in: Blum/Krüger, Wissenschaftsrecht und Wissenschaftspolitik in nationaler und europäischer Sicht, WissR Beiheft 11, 1993 , S. 81 (85ff.). 
Zustimmung des beliehenen Rechtssubjekts bedarf, die DFG jedoch öffentlich-rechtliche Bindungen für ihre Tätigkeit ausdrücklich ablehnt.

Daher sollen die Mittlerorganisationen bei der Vergabe der Mittel nach h. M. in privatrechtlicher Form in Wahrnehmung eigener satzungsgemäßer Aufgaben handeln. ${ }^{25}$ Ihr Verhalten sei demnach allein privatrechtlich zu bewerten. ${ }^{26}$ Wäre indes der Endzweck der Zuwendung die Förderung des Privaten, der selbst das gewünschte öffentliche Ziel nicht erreichen kann, würde dies ein unzulässiges staatliches Geschenk darstellen. ${ }^{27}$ Aus diesem Grund wird vertreten, die Mittlerorganisationen erhielten das Geld nicht nur, um es an Dritte weiterzuleiten, sondern ebenso, um eine plurale Förderlandschaft zu erhalten. ${ }^{28}$ Dass dies allerdings wenn überhaupt nur ein unbedeutender Nebenzweck ist, wird daran deutlich, dass die Anzahl der Mittlerorganisationen in den einzelnen wissenschaftlichen Tätigkeitsfeldern stark begrenzt ist. Die Förderung der Mittlerorganisation ist also lediglich ein notwendiger Zwischenschritt zur Erreichung eines öffentlichen Zwecks. Der Private nimmt damit nicht nur eine eigene Aufgabe wahr. Allein der mit der Förderung des Privaten (mittelbar) verfolgte öffentliche Zweck ist allerdings nicht ausreichend, um die Weiterleitung der Mittel dem öffentlichen Recht zu unterwerfen. Dies würde zu einer übermäßigen Ausdehnung des öffentlichen Rechts auf anerkanntermaßen ausschließlich privatrechtliche Sachverhalte führen, wie z. B. der Erwerb von Maschinen durch Subventionsgelder. Es ist also möglich, dass die Weiterleitung der Mittel durch Mittlerorganisationen ausschließlich privatrechtlich einzustufen ist.

Zweifelhaft ist, ob zwischen diesen rein öffentlich-rechtlichen und privatrechtlichen Beteiligungsformen noch eine Mischform existiert, konkret dass der Private zwar umfassend die Durchführung eines staatlichen Förderprogramms öffentlich-rechtlich wahrnimmt, ohne jedoch dazu öffentlich-rechtliche Handlungsbefugnisse zu nutzen. Das setzt voraus, dass eine staatliche Aufgabe ausschließlich privatrechtlich erledigt werden kann. Damit ist die Frage der Zulässigkeit der Privatisierung von Verwaltungsaufgaben aufgeworfen. Ob bei einer fehlenden ausdrücklichen gesetzlichen Regelung wie im Falle der Wissenschaftsförderung eine Wahlfreiheit in Bezug auf die verwendeten rechtlichen Instrumente besteht, ist umstritten. Teilweise wird diese generell abgelehnt. ${ }^{29}$ Das öffentliche Recht sei ein zwingendes Sonderrecht des Staates, die Geltung des Privatrechts daher ausgeschlossen. ${ }^{30}$ Die Annahme dieser Sonderrechtslehre, öffentliches Recht sei ein zwingendes Sonderrecht des Staates, kann indes in dieser Allgemeinheit nicht geteilt werden. Der zwingende Charakter muss vielmehr für jede Gesetzesnorm separat bestimmt werden. Die differenzierende Zweistufentheorie geht im Gegensatz dazu von einer Formenwahlfreiheit der Verwaltung aus. Jedenfalls die Entscheidung über das „Ob“ der Leistungsgewährung sei aber öffentlich-rechtlich. ${ }^{31}$ Nach der dritten Ansicht

25 Dittrich/Lamm (o. Fußn. 15), § 44 BHO Tz. 79.13; Dommach (o. Fußn. 15), § 44 BHO Rn. 61.

26 Dittrich/Lamm (o. Fußn. 15), § 44 BHO Tz. 80.2.

27 Vgl. Berg, GewArch 1987, 1 (2); Krämer/Schmidt, Zuwendungsrecht, 2007, B III S. 2 f.

28 Streiter (o. Fußn. 19), S. 327 f.

29 Pestalozza, Formenmissbrauch des Staates, 1973, S. 166ff., 185; Kempen, Die Formenwahlfreiheit der Verwaltung, 1989, S. 109ff., 122.

30 Pestalozza (o. Fußn. 29), S. 174ff.

31 VGH Kassel, NVwZ 2003, 238 (238); OVG Münster, NVWZ-RR 2006, 223 (223). 
besteht bei der staatlichen Leistungsverwaltung freie Formwahl. ${ }^{32}$ Hiergegen spricht auf den ersten Blick, dass sich der Staat dadurch seiner grundrechtlichen Bindung entziehen kann. Um das zu verhindern, gilt dann allerdings das Verwaltungsprivatrecht. ${ }^{33}$ Damit wird dem Zweck der Zweistufentheorie, eine Grundrechtsfreiheit bei der staatlichen Mittelvergabe zu verhindern, ${ }^{34}$ auch von der dritten Ansicht Rechnung getragen. Entscheidendes Argument zur Ablehnung der Zweistufentheorie ist jedoch, dass sie die Pflicht voraussetzt, über das Bestehen subjektiv-öffentlicher Rechte müsse durch Verwaltungsakt unter Ausschluss vertraglicher Handlungsformen entscheiden werden. ${ }^{35}$ Überzeugende Argumente für eine solche sind nicht ersichtlich. ${ }^{36}$ Somit existiert mit dem Verwaltungsprivatrecht zwischen den rein öffentlich-rechtlichen und privatrechtlichen Beteiligungsformen eine Mischform.

\section{Abgrenzungskriterien zwischen Privatrecht und öffentlichem Recht}

Die Feststellung eines gemischten Bereichs löst die Problematik der Abgrenzung zwischen öffentlichem und Privatrecht nicht. Es bleibt die Frage, an welchem Punkt eine Organisation den Bereich des rein privaten bzw. rein öffentlichen verlässt. Als Abgrenzungskriterium kommt zunächst das der Rechtsform in Betracht. Allein eine privatrechtliche Rechtsform von Organisationen führt aber nicht dazu, dass diese und ihr Verhalten zwingend als ausschließlich privatrechtlich einzuordnen sind. ${ }^{37}$ Die Rechtsfigur der Beleihung macht deutlich, dass Private vollständig staatlich einverleibt werden können und dann folgerichtig dem öffentlichen Recht unterfallen. Auch die Verwendung privatrechtlicher Handlungsformen zur Zielerreichung schließt eine Anwendbarkeit des öffentlichen Rechts nicht von vornherein aus. Die verfassungsrechtlich gebotene Entwicklung des Verwaltungsprivatrechts hat deutlich gemacht, dass eine Flucht von grundrechtsgebundenen Organisationen ins Privatrecht keine Privatautonomie schafft. ${ }^{38}$ Die wahrgenommene Aufgabe ist als Abgrenzungskriterium ebenfalls weitgehend ungeeignet. Öffentliche Zwecke werden nicht exklusiv mit staatlichen Mitteln und öffentlich-rechtlichen Organisationsformen verfolgt. ${ }^{39}$ Bei Aufgaben, die traditionell sowohl staatlich als auch privat durchgeführt werden, was bei der Wissenschaftsförderung der Fall ist, tendiert der Zuordnungswert der Aufgabe sogar gegen Null. ${ }^{40}$

Einzig sinnvolles Abgrenzungskriterium ist daher der Grad der Entfernung vom Staatskörper. ${ }^{41}$ Entscheidend ist also die Steuerbarkeit des Privaten durch die Regierung

32 Ehlers, in: Schoch/Schmidt-Aßmann/Pietzner, VwGO 2008, § 40 Rn. 255ff.

33 Ehlers (o. Fußn. 32), § 40 Rn. 259.

34 Vgl. Stelkens, Verwaltungsprivatrecht, 2005, S. 968; Ehlers (o. Fußn. 32), § 40 Rn. 259.

35 Stelkens (o. Fußn. 34), S. 973.

36 Vgl. ausführlich dazu Stelkens (o. Fußn. 34), S. 973.

37 So im Hinblick auf die DFG Salaw-Hanslmaier, Die Rechtsnatur der Deutschen Forschungsgemeinschaft, 2003, S. 96.

38 Vgl. Schuppert, Die Erfüllung öffentlicher Aufgaben durch verselbständigte Verwaltungseinheiten, 1981, S. 81.

39 Schuppert (o. Fußn. 38), S. 87.

40 Schuppert (o. Fußn. 38), S. 188; ähnlich im Hinblick auf die Mittlerorganisationen Streiter (o. Fußn. 19), S. 330.

41 Schuppert (o. Fußn. 38), S. 168. 
bzw. Verwaltung. Dabei soll es unerheblich sein, ob diese auf rechtlichen Befugnissen oder tatsächlichen Umständen beruht. ${ }^{42}$ Im Ergebnis müsse der Staat aber erheblichen ${ }^{43}$ bzw. beherrschenden ${ }^{44}$ Einfluss auf die Verteilung der Mittel besitzen. Ein solcher resultiere insbesondere aus dem Umfang der Zweckbestimmung der bereitgestellten Mittel und der Beteiligung des Staates an den über die Verteilung entscheidenden Gremien. ${ }^{45}$ Dieser Verweis auf die Finanzierung ist zutreffend, da die Privaten auf diese angewiesen sind, um die von ihnen angestrebte Wissenschaftsförderung zu realisieren. Ohne dieses Abhängigkeitsverhältnis ist ein beherrschender staatlicher Einfluss gar nicht denkbar. Die Finanzierung ist aber keine hinreichende Bedingung für die Abhängigkeit Privater von staatlichen Instanzen. ${ }^{46}$ Ansonsten würde es zu einer weitgehenden Verstaatlichung wirtschaftlich nicht rentabler Bereiche kommen. ${ }^{47}$ Zwar kann über die im Rahmen der Finanzierung erfolgende Zwecksetzung Einfluss auf die Förderentscheidung ausgeübt werden, ${ }^{48}$ indes kann durch solche abstrakten Vorgaben nur eine beschränkte Prägung der endgültigen Förderentscheidung vorgenommen werden. Die globale Einstufung, eine hälftige Finanzierung sei für einen beherrschenden Einfluss des Staates ausreichend, ${ }^{49}$ geht deshalb zu weit. Es müssen daher weitere Merkmale hinzutreten. Als solches wird von Streiter die Grundrechtsrelevanz des geförderten Bereichs befürwortet, wodurch die Wirkung der Gelder als Maßstab genommen wird. ${ }^{50}$ Auch wenn sich der Staat durch die Aufgabenübertragung an Private nicht seiner grundrechtlichen Schutzpflichten entziehen kann, würde dadurch eine nicht erforderliche Verstaatlichung privater Bereiche erfolgen. Der Staat trägt durch die Finanzierung der Mittlerorganisation zu öffentlichen Zwecken eine Gewährleistungspflicht, aufgrund derer er bei einem zweckwidrigen Mitteleinsatz auf den Zuwendungsempfänger einwirken oder dessen Finanzierung beenden muss. Es bleibt infolgedessen dabei, dass es ausschließlich aufgrund des konkreten staatlichen Einflusses möglich ist, private Organisationen öffentlich-rechtlichen Bindungen zu unterwerfen. Dieser ergibt sich insbesondere aus der personellen Verflechtung von Staat und Privatem, ${ }^{51}$ vor allem aus der Mitgliedschaft von staatlichen Vertretern im für die Förderentscheidung zuständigen Gremium. ${ }^{52}$ Abgrenzungskriterien sind also zum einen der Umfang und die Modalitäten der Finanzierung der Mittlerorganisation durch den Staat und zum anderen der Einfluss der staatlichen Vertreter in den Gremien der Mittlerorganisation.

42 Schuppert (o. Fußn. 38), S. 169.

43 Trute, Forschung zwischen grundrechtlicher Freiheit und staatlicher Institutionalisierung, 1994, S. 679 f.; Salaw-Hanslmaier (o. Fußn. 37), S. 151; ähnlich BGH, NJW 2003, 2451 (2452).

44 Streiter (o. Fußn. 19), S. 295.

45 Trute (o. Fußn. 43), S. 679.

46 Schuppert (o. Fußn. 38), S. 171; Salaw-Hanslmaier (o. Fußn. 37), S. 153; ähnlich Streiter (o. Fußn. 19), S. $331 \mathrm{f}$.

47 Salaw-Hanslmaier (o. Fußn. 37), S. 159.

48 Salaw-Hanslmaier (o. Fußn. 37), S. 153.

49 Ehlers, Verwaltung in Privatrechtsform, 1984, S. 12 f.

50 Streiter (o. Fußn. 19), S. 338 f.

51 Schuppert (o. Fußn. 38), S. 189; Salaw-Hanslmaier (o. Fußn. 37), S. 154.

52 Streiter (o. Fußn. 19), S. 333 f. 


\section{Einordnung der Förderverfahren der DFG in die Rechtsgebietsdogmatik}

Zur Einordnung der DFG-Förderverfahren ist demnach zunächst festzustellen, dass die DFG von Bund und Länder sowohl eine institutionelle als auch eine projektbezogene Förderung erhält. Fast 80 Prozent der Einnahmen der DFG stammen aus der institutionellen Förderung durch Bund und Länder. ${ }^{53}$ Deren Gegenstand ist nicht ein einzelnes abgegrenztes Vorhaben, sondern die DFG als solche. ${ }^{54}$ Die Gelder dienen nach den Erläuterungen im Haushaltsplan des Bundes der Wissenschaftsförderung, wobei die unterschiedlichen Programme der DFG, insbesondere das Normalverfahren, die Sonderforschungsbereiche und die Graduiertenkollegs erwähnt werden. ${ }^{55}$ Nichtsdestotrotz sind dies nur Erläuterungen, keine verbindlichen Beschränkungen der Mittelverwendung. Der für die Annahme einer staatlichen Aufgabe bei der Weiterleitung der Gelder an die Endempfänger notwendige erhebliche Einfluss kann deshalb lediglich aus der Mitgliedschaft von Staatsvertretern in den Entscheidungsgremien der DFG resultieren. Ein ausreichender Einfluss soll jedenfalls dann vorliegen, wenn der Staat in diesen Gremien eine Förderung verhindern kann. ${ }^{56}$ Dies wird überzeugend damit begründet, dass in einer solchen Konstellation eine Förderung aus politischen Gründen unterbleiben kann. ${ }^{57}$ Bund und Länder stellen 32 der 73 Mitglieder des Hauptausschusses der DFG, welcher über die Anträge auf Förderung im Normalverfahren mit einfacher Mehrheit entscheidet. Der Staat kann also in diesen Verfahren weder gegen den Willen der DFGVertreter eine Förderung durchsetzen noch diese verhindern, so dass die Entscheidungen im Normalverfahren als rein privatrechtlich einzustufen sind. Die h. M. geht darüber hinaus sogar generell von begrenzten staatlichen Einflussmöglichkeiten aus und sieht die Weiterleitung der Gelder durch die DFG in keinem Fall als Ausübung von Staatsgewalt an. ${ }^{58}$ Betont wird, dass die DFG durch ihre organisatorische Ausgestaltung eine starke Verwurzelung im nicht staatlichen Bereich besitze. ${ }^{59}$ Dabei wird indes verkannt, dass die staatlichen Vertreter in den Bewilligungsausschüssen der Sonderforschungsbereiche und Graduiertenkollegs die Hälfte der Stimmen und damit eine Speerminorität besitzen. Die staatlichen Einflussmöglichkeiten in einer solchen Konstellation sind so umfangreich, dass die Vergabeentscheidungen in diesen Förderbereichen als Ausübung von Staatsgewalt einzustufen sind. ${ }^{60}$ Gleiches gilt im Ergebnis für die Exzellenzinitiative. Zwar haben die staatlichen Vertreter mit 32 von 71 Stimmen im Bewilligungsausschuss keine Speerminorität ( $\$ 4$ Abs. 3, 8 ExV). Allerdings wird dieses nicht ausschlaggebende staatliche Stimmengewicht durch das zweite Kriterium, die Zweckbestimmung der Mittel aufgewogen. So macht bereits die Zweckbestimmung im Haushaltsplan ${ }^{61}$ deutlich, dass die Zuweisung der Mittel an die DFG zur Weiterleitung nach

53 Insgesamt decken Bund und Länder 99,7 \% des Etats der DFG.

54 Vgl. Krämer/Schmidt (o. Fußn. 27), C III S. 2.

55 Vgl. Bundeshaushaltsplan Kap. 3003 Titelgruppe 30.

56 Trute (o. Fußn. 43), S. 684.

57 Trute (o. Fußn. 43), S. 684.

58 Dittrich/Lamm (o. Fußn. 15), § 44 BHO Tz. 80.2; Dommach (o. Fußn. 15), § 44 BHO Rn. 61; a. A. Salaw-Hanslmaier (o. Fußn. 37), S. 216.

59 Schuppert (o. Fußn. 38), S. 181; differenzierend Salaw-Hanslmaier (o. Fußn. 37), S. 161, wonach die DFG eine typische Einrichtung des Halbstaatlichen ist.

60 Trute (o. Fußn. 43), S. 684 f.

61 Vgl. Bundeshaushaltsplan Kap. 3003 Titel 685 04-139. 
Maßgabe der Bund-Länder-Vereinbarung über die Exzellenzinitiative erfolgt. Aufgrund dessen sind der Kreis der Antragsteller und förderungsfähigen Projekte, die Auswahlkriterien und der Umfang der Zuweisungen an die Endempfänger vorgegeben. Zwar verbleiben noch Entscheidungsspielräume, insbesondere bei den Kriterien zur Feststellung der wissenschaftlichen Exzellenz. Diese sind aber deutlich geringer als im Rahmen der institutionellen Förderung, weshalb die DFG auch mit der Durchführung dieses Förderprogramms Staatsgewalt ausübt.

\section{Ansprüche der Antragsteller in den Förderverfahren der DFG}

Soweit die Durchführung der Förderverfahren der DFG Ausübung von Staatsgewalt darstellt, löst dies öffentlich-rechtliche Bindungen aus, ${ }^{62}$ führt aber nicht zwingend dazu, dass eine Förderentscheidung als Verwaltungsakt einzustufen ist. ${ }^{63}$ Aufgrund der öffentlich-rechtlichen Bindungen unterliegt die DFG bei der Ausführung der koordinierten Programme allerdings dem Verwaltungsprivatrecht. ${ }^{64}$ Dieses schränkt die rechtsgeschäftliche Autonomie ein, indem es die privatrechtlichen Regelungen durch öffentlich-rechtliche ergänzt, überlagert oder modifiziert. ${ }^{65}$ Unstreitig ist die daraus resultierende Bindung an die Grundrechte. ${ }^{66}$ Aus diesem Grund ist die DFG verpflichtet, das Rechtsverhältnis zu den Hochschulen so auszugestalten, dass nach Zweck, Inhalt und Umfang der Förderung der Empfänger seine Rechtsposition ungeschmälert durchsetzen kann. ${ }^{67}$ Inwieweit darüber hinaus einfachgesetzliche öffentlich-rechtliche Normen anwendbar sind, ist dagegen umstritten. Eine unmittelbare Anwendung des VwVfG scheidet aus, da nach $\S 1$ VwVfG nur öffentlich-rechtliche Tätigkeiten erfasst sind. ${ }^{68}$ Einer analogen Geltung des VwVfG ${ }^{69}$ ist die h. M. zutreffend nicht gefolgt. ${ }^{70}$ Aus der Entstehungsgeschichte des VwVfG ergibt sich, dass der Gesetzgeber bewusst auf die Einbeziehung privatrechtlicher Tätigkeiten durch Behörden verzichtet hat. ${ }^{71}$ Anwendbar sind Vorschriften des VwVfG deshalb ausschließlich, wenn sie in ihrer konkreten Ausgestaltung Ausdruck eines allgemeinen Rechtsgedankens oder einfach-gesetzliche Konkretisierungen des Verfassungsrechts sind. ${ }^{72}$

62 Trute (o. Fußn. 43), S. 685.

63 Vgl. dazu auch $B G H$, NJW 2000, 1042 (1042 f.).

64 Nach Ansicht von Salaw-Hanslmaier (o. Fußn. 37), S. 219 unterliegt die DFG sogar generell dem Verwaltungsprivatrecht.

65 Wolff/Bachof/Stober/Kluth, Verwaltungsrecht, 12. Aufl. 2007, § 23 Rn. 62.

66 Kopp/Ramsauer, VwVfG, 10. Aufl. 2008, Einführung Rn. 51; Wolff/Bachof/Stober/Kluth (o. Fußn. 65), § 23 Rn. 64 m. w. N.

67 Streiter (o. Fußn. 19), S. 15.

68 Kopp/Ramsauer (o. Fußn. 66), Einführung Rn. 51 a; Schmitz, in: Stelkens/Bonk/Sachs, VwVfG, 7. Aufl. 2008, § 1 Rn. 116.

69 Zezschwitz, NJW 1983, 1873 (1881).

70 BGH, NJW 2003, 2451 (2453); Schmitz (o. Fußn. 68), § 1 Rn. 116 f.; Kopp/Ramsauer (o. Fußn. 66), Einführung Rn. 51 a.

71 BGH, NJW 2003, 2451 (2453); Schmitz (o. Fußn. 68), §1 Rn. 117; Wolff/Bachof/Stober/ Kluth (o. Fußn. 65), § 23 Rn. 68.

$72 B G H$, NJW 2003, S. 2451 (2453). 


\section{Recht auf Akteneinsicht}

Dass die Förderverfahren der DFG Defizite in der Transparenz aufweisen, zeigt nicht nur der Fall von Harro Lentz. Selbst in den eigenen Befragungen der DFG und des Wissenschaftsrates über die Klarheit des Verfahrens der Exzellenzinitiative aus Sicht der Hochschulen wurden nur mäßige Bewertungen abgegeben. ${ }^{73}$ Insbesondere gewährt die DFG den Antragstellern keine Einblicke in die Gutachten, die der Förderentscheidung zugrunde liegen. Dieser Umstand macht es relevant, ob und inwieweit die DFG verpflichtet ist, Informationen herauszugeben.

\section{a) Informationspflichten nach dem IFG}

Eine Verpflichtung der DFG, Unterlagen über die Entscheidungsprozesse herauszugeben, kommt zunächst nach $\S 1$ Abs. $1 \mathrm{~S} .1$ IFG in Betracht. Das IFG fordert dafür keine eigene Betroffenheit des Antragstellers. Anspruchsverpflichtet sind gemäß $\S 1$ Abs. 1 S. 1 IFG die Behörden des Bundes, wobei der Behördenbegriff dem des $\S 1$ Abs. 4 VwVfG entspricht. ${ }^{74}$ Zwar erfüllt die DFG bei der Durchführung des Vergabeverfahrens diesen mangels Beleihung nicht. Allerdings gewährt $\S 1$ Abs. 1 S. 3 IFG den Informationszugang auch in den Fällen, in denen sich die öffentliche Hand zur Erfüllung ihrer Aufgaben Privater bedient, ohne diese zu beleihen. Indes wird dadurch der Private nicht unmittelbar zur Auskunftserteilung verpflichtet. Vielmehr bleibt die den Privaten zur Aufgabenerfüllung nutzende Behörde gemäß $§ 7$ Abs. 1 S. 2 IFG den Anspruchsberechtigten gegenüber verpflichtet. Dies wird teilweise so verstanden, dass nur Informationen über die Aufgabenerfüllung erfasst sind, die sich bereits bei der Behörde befinden. ${ }^{75}$ Dabei wird indes verkannt, dass $\S 7$ Abs. 1 S. 2 IFG lediglich klarstellt, dass, wenn sich eine Behörde zur Erfüllung ihrer öffentlich-rechtlichen Aufgaben Privater bedient, sie selbst Anspruchsgegnerin bleibt und keine unmittelbaren Informationsansprüche gegenüber Privaten entstehen. ${ }^{76}$ Aussagen über den Umfang des Informationsanspruchs können der Regelung folglich nicht entnommen werden. § 1 Abs. $1 \mathrm{~S} .3$ IFG stellt vielmehr klar, dass eine Verlagerung der Aufgabenerfüllung ins Privatrecht, den Informationsanspruch nicht verringert. Deshalb wird aus $\S 1$ Abs. 1 S. 3 IFG zutreffend eine Verpflichtung des Privaten im Innenverhältnis zur Behörde abgeleitet, die Informationen bereitzustellen. ${ }^{77}$

Erfasst werden durch $\S 1$ Abs. 1 S. 3 IFG vornehmlich Verwaltungshelfer, aber auch Private, die selbständig, jedoch im Gegensatz zu Beliehenen nicht hoheitlich zur Erfüllung öffentlicher Aufgaben tätig werden. ${ }^{78}$ Eine solche Tätigkeit nimmt die DFG wie bereits erläutert wahr. Des Weiteren muss sich die Behörde des Privaten bedienen. Das

73 Wissenschaftsrat, Bericht der Strategiekommission des Wissenschaftsrates: Auswertung der geförderten Zukunftskonzepte, Drs. 8815-08, 2008, S. 50ff.

74 Schmitz/Jastrow, NVwZ 2005, 984 (987); Rossi, Informationsfreiheitsgesetz, 2006, § 1 Rn. 35, 40.

75 Jastrow/Schlatmann, Informationsfreiheitsgesetz, 2006, § 7 Rn. 17.

76 BT-Drs. 15/4493, S. 14.

77 Rossi (o. Fußn. 74), § 1 Rn. 71; Schoch, Informationsfreiheitsgesetz, 2009, § 1 Rn. 31.

78 Rossi (o. Fußn. 74), § 1 Rn. 74; Schoch (o. Fußn. 77), § 1 Rn. 117. 
wäre unzweifelhaft der Fall gewesen, wenn das BMBF der DFG den Auftrag erhalten hätte, die koordinierten Förderverfahren durchzuführen. Da die vertraglichen Vereinbarungen der DFG mit dem BMBF nicht veröffentlicht sind, kann dies nicht sicher angenommen werden. Jedenfalls gebietet aber der Gesetzeszweck die (analoge) Anwendbarkeit des IFG auf die Tätigkeit der DFG im Rahmen der koordinierten Förderverfahren. So dient $\S 1$ Abs. 1 S. 3 IFG dazu den Anspruch auf Informationszugang umfassend auszugestalten. ${ }^{79}$ Eine „Flucht ins Privatrecht“ soll den Informationsanspruch nicht aufheben, so dass die DFG nach $\S 1$ Abs. 1 S. 3 IFG (analog) mittelbar anspruchsverpflichtet ist. Die weiteren Anspruchsvoraussetzungen des IFG sind ebenfalls erfüllt, da keiner der Ausschlussgründe nach $\S \S 3 \mathrm{ff}$. IFG in Betracht kommt. Die Antragsteller in koordinierten Verfahren können demnach gegenüber dem BMBF Einsicht in die Akten und Protokolle der DFG verlangen.

\section{b) Informationspflichten nach dem VwVfG}

Nach $§ 29$ Abs. 1 S. 1 VwVfG hat die Behörde den Beteiligten Einsicht in die das Verfahren betreffenden Akten zu gestatten, soweit deren Kenntnis zur Geltendmachung oder Verteidigung ihrer rechtlichen Interessen erforderlich ist. Ob diese Vorschrift bei privatrechtlicher Erledigung staatlicher Aufgaben analog anwendbar ist, ist umstritten. Teilweise wird dies abgelehnt. Zwar bestehe ein (berechtigtes) Bedürfnis nach Akteneinsicht, diesem werde aber durch das IFG genüge getan. ${ }^{80}$ Des Weiteren unterlaufe das Akteneinsichtsrecht die Anonymität der Gutachter. In diesem Fall werde die Bereitschaft zu einer solchen Tätigkeit sinken, was die Funktionsfähigkeit des gesamten Begutachtungsverfahrens gefährde. Aufgrund dieser Besonderheit gelte $§ 29 \mathrm{VwVfG}$ nicht. ${ }^{81}$ Diese Einschätzung der Wirkungen eines transparenten Verfahrens, welche auch die DFG vertritt, ${ }^{82}$ ist nicht unumstritten. Als Vorteil der Anonymität wird genannt, dass sich die Gutachter nicht mit Kritik zurückhalten, da sie keine Sanktionen zu befürchten haben. ${ }^{83}$ Dies sei notwendig, weil nur so die aufgrund begrenzter finanzieller Mittel notwenige hohe Abweisungsquote erreicht werden könne. ${ }^{84} \mathrm{Da}$ die Anonymität der Gutachter vor allem einen Schutz vor den „Mächtigen im Fach“ darstelle, würde deren Aufhebung die Wahrscheinlichkeit, dass die Statusschwächeren im Fach diskriminiert werden, erhöhen. ${ }^{85}$ Gleichzeitig würde dadurch die Tendenz unterstützt, in der Begutachtung inhaltlich den "Mainstream" zu bedienen und die riskante Förderung des Kreativ-Ungewöhnlichen zu scheuen. ${ }^{86}$ Als Argument für die Offenlegung der Gutachten wird angeführt, dass im Rechtsstaat grundsätzlich alle wichtigen Entscheidungen transparent sein müssen. ${ }^{87}$ Durch die Aufhebung der Anonymität könne die Entwick-

79 BT-Drs. 15/4493, S. 8.

80 Bonk/Kallerhoff, in: Stelkens/Bonk/Sachs, VwVfG, 7. Aufl. 2008, § 29 Rn. 14.

81 Salaw-Hanslmaier (o. Fußn. 37), S. 241; a. A. Streiter (o. Fußn. 19), S. 374.

82 www.spiegel.de/wissenschaft/mensch/0,1518,394373,00.html (Stand: 11.8.2009).

83 Smith, in: Wager/Godlee/Jefferson, Erfolg im Peer Review, 2007, S. 115 (127); Fletcher/

Fletcher, in: Wager/Godlee/Jefferson, Erfolg im Peer Review, 2007, S. 23 (31).

84 Smith (o. Fußn. 83), S. 128.

85 Neidhardt, in: Hornbostel/Simon, Wie viel (In-)Transparenz ist notwendig, 2006, S. 7 (8).

86 Neidhardt (o. Fußn. 85), S. 8.

87 Smith (o. Fußn. 83), S. 127. 
lung geeigneter Kontrollverfahren in Folge der Erhöhung der Diskursivität der Beurteilungen gefördert werden. ${ }^{88}$ Auch kämen Befangenheit oder Konflikte der Gutachter eher ans Licht, wenn die Autoren die Identität des Gutachters kennen. ${ }^{89}$ Daneben sollen sich die Gutachter bei fehlender Anonymität mehr Mühe bei der Erstellung der Gutachten geben..$^{90}$

Dazu ist zusammenfassend zunächst festzustellen, dass negative Wirkungen der Transparenz im Hinblick auf die Bereitschaft bestehen, als Gutachter tätig zu werden. ${ }^{91}$ Dagegen ist der Effekt der namentlichen Begutachtung auf die Qualität der Begutachtung noch nicht abschließend geklärt. Die vorliegenden Studien geben diesbezüglich kein konsistentes Bild. ${ }^{92}$ Nachteile für das Begutachtungsverfahren durch die Erhöhung der Transparenz können deshalb nicht vollständig ausgeschlossen werden. Sie erscheinen allerdings nicht zwingend größer als die im Gegenzug erzielbaren positiven Effekte. Hinzu kommt, dass die Transparenz aus rechtsstaatlichen Erwägungen geboten ist. Andernfalls haben die Betroffenen praktisch keine Chance, ihre Rechte wirksam wahrzunehmen. ${ }^{93}$ Infolgedessen ist $§ 29 \mathrm{VwVfG}$ im Rahmen der koordinierten Förderprogramme der DFG anwendbar.

\section{Begründungspflicht und-umfang}

Im Hinblick auf die Exzellenzinitiative hat die DFG selbst eingeräumt, dass es Unzufriedenheiten bei den Hochschulen über die Qualität der Begründungen gegeben hat. ${ }^{94}$ Neben negativen Auswirkungen auf die Akzeptanz des Verfahrens besteht dadurch die Gefahr, dass aufgrund von generellen Nachweisproblemen in aufwendigen Verfahren eine glaubhafte Geltendmachung von Fehlern unmöglich wird. Die Begründung ist also für die Beweisführung von essentieller Bedeutung, weshalb die h. M. aus dem Rechtsstaatsprinzip die Anwendung von $\S 39$ VwVfG zutreffend ableitet. ${ }^{95}$ Die ablehnenden Vergabeentscheidungen der DFG im Rahmen der koordinierten Förderverfahren bedürfen daher nach entsprechender Heranziehung von §39 Abs. 1 S. 1 VwVfG einer Begründung. Dass dieses Erfordernis nicht nur für eingreifende Verwaltungsakte, sondern generell für sämtliche belastenden Maßnahmen der Staatsgewalt gilt, folgt jedenfalls aus der grundrechtlichen Verankerung der Begründungspflicht. ${ }^{96}$

88 Hornbostel/Simon, in: dies., Wie viel (In-)Transparenz ist notwendig, 2006, S. 5 (5).

89 Godlee/Dickersin, in: Wager/Godlee/Jefferson, Erfolg im Peer Review, 2007, S. 51 (79).

90 Fletcher/Fletcher (o. Fußn. 83), S. 31.

91 Hansen, in: Hornbostel/Simon, Wie viel (In-)Transparenz ist notwendig, 2006, S. 37 (40).

92 Godlee/Dickersin (o. Fußn. 89), S. 65.

93 Streiter (o. Fußn. 19), S. 371 f.

94 DFG/Wissenschaftsrat, Bericht der Gemeinsamen Kommission zur Exzellenzinitiative an die Gemeinsame Wissenschaftskonferenz, 2008, S. 20.

95 Kopp/Ramsauer (o. Fußn. 66), § 39 Rn. 4 m. w. N.; Streiter (o. Fußn. 19), S. 360, 364; SalawHanslmaier (o. Fußn. 37), S. 240; a. A. Liebetanz, in: Obermayer, VwVfG, 3. Aufl. 1999, $\S 39$ Rn. 6, 12.

96 Ausführlich U. Stelkens, in: Stelkens/Bonk/Sachs, VwVfG, 7. Aufl. 2008, § 39 Rn. 2; konkret im Hinblick auf die Förderverfahren der DFG Trute (o. Fußn. 43), S. 690. 
$\mathrm{Zu}$ betonen ist, dass der Begründungszwang ebenfalls für Kollegialentscheidungen ${ }^{97}$ und bei Vorliegen eines Beurteilungsspielraums gilt. ${ }^{98}$ Wichtig für den Antragsteller ist dabei nicht nur, dass sie über die Nichtauswahl informiert werden. Allein dies ermöglicht bei begrenzten Kapazitäten generell keine Einschätzung, ob die Nichtauswahl einen Rechtsverstoß darstellt. Notwendig ist im Hinblick auf das Rechtsstaatsprinzip und den Grundrechtsschutz die Nennung der erfolgreichen Bewerber und deren Qualitäten, die es rechtfertigen, sie den anderen Bewerbern vorzuziehen. ${ }^{99}$ Zudem dürfen die Begünstigungen erst nach Ablauf einer angemessenen Frist endgültig gewährt werden. ${ }^{100}$

\section{Materielle Anforderungen an die Vergabeentscheidungen der DFG}

Bei den materiellen Anforderungen an die Vergabeentscheidungen stellt sich die Frage, ob die Antragsteller die inhaltliche Richtigkeit der Entscheidungen überprüfen lassen können oder ob ihnen lediglich ein faires Verfahren garantiert ist. Die h. M. vertritt letzteres und nimmt daher lediglich einen Schutz vor einer willkürlichen Mittelvergabe an. ${ }^{101}$ Einerseits wird dies damit begründet, die Gelder der DFG seien bloße Ergänzungsmittel, auf die Wissenschaftler und Hochschulen nicht angewiesen seien. ${ }^{102}$ Angesichts der Verlagerung der finanziellen Ressourcen von der Grundausstattung zu den Drittmitteln und des gleichzeitig steigenden Finanzbedarfs der Wissenschaft, kann dies aber nicht überzeugen. Des Weiteren wird die Reduzierung des Kontrollumfangs mit der Sachnähe der Gutachter gerechtfertigt. So gut wie alle Vergabeentscheidungen erforderten eine hohe fachspezifische Expertise, die sich über die gesamte Brandbreite der wissenschaftlichen Disziplinen erstrecke. ${ }^{103}$ Den Richtern fehle es daher an den notwendigen Kenntnissen, um die Qualität von Forschungsanträgen in den unzähligen Wissenschaftsdisziplinen zu beurteilen. Eine gerichtliche Überprüfung würde deshalb am nicht sinnvoll auslösbaren Gutachterstreit scheitern. ${ }^{104}$ Aus diesem Grund wird ein Beurteilungsspielraum der DFG angenommen. ${ }^{105}$

Beurteilungsspielräume stehen jedoch in einem Spannungsverhältnis zu Art. 19 Abs. 4 GG, der grundsätzlich eine vollständige gerichtliche Kontrolle in rechtlicher und tatsächlicher Hinsicht verlangt. ${ }^{106}$ Allerdings ist bereits die Anwendbarkeit dieser Vorschrift auf die Förderverfahren der DFG zweifelhaft, da die Frage, ob Tätigkeiten des

97 OVG Magdeburg, LKV 1994, 60 (62); Kopp/Ramsauer (o. Fußn. 66), § 39 Rn. 7; Dolzer, DÖV 1985, 9 (16ff.).

98 VG Berlin, NJW 1973, 1148 (1148); U. Stelkens (o. Fußn. 96), § 39 Rn. 8.

99 Vgl. U. Stelkens (o. Fußn. 96), § 39 Rn. 20.

100 U. Stelkens (o. Fußn. 96), § 35 Rn. 161.

101 Streiter (o. Fußn. 19), S. 218 f.; Salaw-Hanslmaier (o. Fußn. 37), S. 229 f.

102 Streiter (o. Fußn. 19), S. 218 f.

103 Streiter (o. Fußn. 19), S. 256.

104 Salaw-Hanslmaier (o. Fußn. 37), S. 229 f.

105 Salaw-Hanslmaier (o. Fußn. 37), S. 231; Streiter (o. Fußn. 19), S. 230, 351.

106 Trute, in: Denninger/Hoffmann-Riem/Schneider/Stein, AK GG, 2007, Art. 33 Abs. 1-3 Rn. 80; ähnlich BVerfGE 61, 82 (111); 88, 40 (56). 
Verwaltungsprivatrechts durch Art. 19 Abs. 4 GG erfasst sind, umstritten ist. ${ }^{107}$ Auch wenn die Anwendbarkeit bejaht wird, schließt Art. 19 Abs. 4 GG Beurteilungsspielräume nicht generell aus. ${ }^{108}$ Ein begrenzter Entscheidungsfreiraum ist zulässig, wenn unbestimmte Rechtsbegriffe wegen der hohen Komplexität und der besonderen Dynamik der geregelten Materie so vage und ihre Konkretisierung im Nachvollzug der Verwaltungsentscheidung so schwierig sind, dass die gerichtliche Kontrolle an die Funktionsgrenzen der Rechtsprechung stößt. ${ }^{109}$ Dies ist $u$. a. bei Beurteilungen und Prognoseentscheidungen im Umweltrecht der Fall, wo aufgrund differierender wissenschaftlicher Ansichten in vielen Fällen selbst durch die Heranziehung von Gutachtern im Gerichtsverfahren die Richtigkeit der behördlichen Entscheidung nicht sicher geklärt werden kann. ${ }^{110}$ Gleiches gilt für Anträge auf Forschungsförderung. Bereits die Bewertung erbrachter wissenschaftlichen Leistungen bereitet enorme Schwierigkeiten. ${ }^{111}$ Das gilt insbesondere für die Qualität wissenschaftlicher Leistungen. Diese ist an den Zahlen einer quantitativen Messung nicht ablesbar. ${ }^{112}$ Noch schwieriger ist die bei der Vergabeentscheidung notwendige Prognose über die zukünftig zu erwartenden wissenschaftlichen Leistungen. Ein Nachvollzug der Vergabeentscheidungen der DFG ist deshalb ohne umfangreiche Spezialkenntnisse nicht möglich. Ein Beurteilungsspielraum begegnet aus diesem Grund im Hinblick auf Art. 19 Abs. 4 GG keinen generellen Bedenken.

Zusätzlich ist aber für das Bestehen eines Beurteilungsspielraums nach h. M. notwendig, dass eine gesetzliche Ermächtigung zur abschließenden Beurteilung existiert, ${ }^{113}$ was im Hinblick auf die Förderverfahren der DFG nicht der Fall ist. Dieser Ansicht ist jedenfalls zuzustimmen, wenn ein Grundrechtseingriff vorliegt. Ob den Hochschulen gemäß Art. 5 Abs. 3GG ein Teilhaberecht an den für alle Hochschulen bereitgestellten Mitteln zusteht, ist umstritten. ${ }^{114}$ Zwar benötigen die Hochschulen die Mittel nicht, um selbst zu forschen oder zu lehren. Sie sind aber dafür verantwortlich, dass die Wissenschaftler die für ihre Arbeit notwendigen Mittel erhalten. Es handelt sich damit bei der Bereitstellung der Mittel an die Hochschulen um Leistungen, welche notwendige Voraussetzung für die Verwirklichung von Grundrechten sind. Hat der Staat bzw. eine vom kontrollierte private Organisation diese bereits anderen Grundrechtsträgern gegenüber erbracht, fordert Art. 5 Abs. 3 GG deshalb eine Gewährung auch zugunsten der übrigen in vergleichbarer Lage befindlichen Anspruchsteller. Die Wissenschaftsfreiheit fungiert damit als spezielles Gleichheitsrecht, welches aufgrund der Be-

107 Bejahend: Schulze-Fielitz, in: Dreier, GG, 2. Aufl. 2004, Art. 19 IV Rn. 53; Huber, in: v. Mangoldt/Klein/Starck, GG, 5. Aufl. 2005, Art. 19 Rn. 426; Schmidt-Aßmann, in: Maunz/ Dürig, GG, 2008, Art. 19 IV Rn. 64; ablehnend Bethge, KritV 1990, 9 (14); Streiter (o. Fußn. 19), S. 231.

108 Vgl. BVerfGE 88, 40 (56); Trute (o. Fußn. 106), Art. 33 Abs. 1-3 Rn. 80.

109 BVerfGE 84, 34 (50).

110 Sachs, in: Stelkens/Bonk/Sachs, VwVfG, 7. Aufl. 2008, § 40 Rn. 212.

111 Vgl. ausführlich zu dieser Problematik Wissenschaftsrat, Bericht der Steuerungsgruppe zur Pilotstudie Forschungsrating Chemie und Soziologie, Drs. 8453-08, 2008.

112 Schmid, Universitätsreform und New Public Management, 2006, S. 11; Seidler, Wissenschaftsmanagement 5/2004, 20 (22); a. A. Münch, Die akademische Elite, 2007, S. 191.

113 Vgl. Maurer, Allgemeines Verwaltungsrecht, 17. Aufl. 2009, § 7 Rn. 33 f. m. w. N.

114 Ablehnend Pernice, in: Dreier, GG, 2. Aufl. 2004, Art. 5 III (Wissenschaft) Rn. 53; bejahend Erichsen/Scherzberg, NVwZ 1990, 8 (17). 
deutung des Art. 5 Abs. 3 GG nicht erst bei willkürlichen, sondern bereits bei unverhältnismäßigen Ungleichbehandlungen verletzt wird. ${ }^{115}$ Ein Beurteilungsspielraum im Rahmen der DFG-Förderverfahren stellt folglich einen Eingriff in das Teilhaberecht der Wissenschaftsfreiheit dar. Mit der h. M. ist deshalb eine gesetzliche Ermächtigung zu fordern, welche nicht vorhanden ist. Die Vergabeentscheidungen der DFG im Rahmen der koordinierten Förderprogramme können folglich im vollen Umfang darauf überprüft werden, ob eine Bestenauslese tatsächlich gelungen ist.

\section{Verletzung der staatlichen Schutzpflicht}

Im Gegensatz zu den koordinierten Förderverfahren gilt im Normalverfahren der DFG das Verwaltungsprivatrecht nicht. Gleichzeitig ist die DFG insbesondere nicht bereit, den Antragstellern die für die Überprüfung der Richtigkeit der Förderentscheidungen notwendige Einsicht in die Unterlagen des Vergabeverfahrens freiwillig zu gewähren. Das BMBF sieht bislang keine Veranlassung, auf die DFG einzuwirken, damit diese Praxis geändert wird. ${ }^{116}$ Diese Untätigkeit erscheint im Hinblick auf die aus Art. 5 III GG folgende Gewährleistungspflicht bedenklich, die insbesondere auch eine finanzielle Förderung der Wissenschaft umfasst. ${ }^{117}$ Die staatliche Förderung der DFG dient der Erfüllung dieser Pflicht, weshalb diese nach Zielrichtung und Wirkungen einer eigenen staatlichen Wissenschaftsförderung vergleichbar ist. Grundrechtsverstöße bei der Fördertätigkeit der DFG sind dem Staat daher zurechenbar, wenn sie intendiert waren oder im geschützten Freiheitsbereich erhebliche Nachteile hervorgerufen haben, die vorhersehbar waren und in Kauf genommen wurden. ${ }^{118}$

Das Teilhaberecht der Wissenschaftler an den zur Wissenschaftsförderung bereitgestellten staatlichen Finanzmitteln ist von zunehmender Bedeutung für die Gewährleistung der Wissenschaftsfreiheit. Gingen früher die Gefahren für diese von staatlichen Publikationsverboten aus, besteht heute die Gefährdung eher darin, dass die für die Wissenschaft notwendigen Ressourcen nicht bereitgestellt werden. Der infolgedessen aus Art. 5 Abs. 3 GG resultierende Anspruch auf eine gleichmäßige Berücksichtigung bei der Förderung, d. h. unter Berücksichtigung der besonderen Situation ihres Aufgabenbereiches angemessen im Vergleich zu den jeweils anderen Hochschullehrern, ${ }^{119}$ kann aufgrund der Schwierigkeiten bei der Festlegung materieller Kriterien nur begrenzt durch diese gesichert werden. Um trotzdem einen angemessenen Grundrechtsschutz zu gewährleisten, ist dieser Mangel an materiellen Vorgaben durch verfahrensrechtliche Sicherungen zu kompensieren. ${ }^{120}$ Diese gewährt die DFG den Antragstellern indes nicht, was den staatlichen Entscheidungsträgern auch bekannt ist. Nicht nur stellt das Verhalten der DFG somit einen Grundrechtseingriff dar. Vielmehr ist dieser dem Staat auch zuzurechnen, der durch seine Nichteinwirkung auf die DFG also seine Gewährleistungspflicht aus Art. 5 Abs. 3 GG verletzt.

115 Vgl. BVerfGE 36, 321 (330 f.); 81, 108 (121).

116 www.spiegel.de/wissenschaft/mensch/0,1518,394373,00.html (Stand: 11.8.2009).

117 BVerfGE 35, 79 (114 f.).

118 Vgl. BVerwGE 71, 183 (193 f.); 90, 112 (121 f.).

119 BVerwGE 52, 339 (348); ähnlich BVerfGE 35, 79 (115).

120 Vgl. zur Bedeutung solcher Mechanismen Trute (o. Fußn. 43), S. 239, 312. 


\section{Gesamtergebnis}

Die zunehmende Leistungsorientierung bei der Mittelvergabe im Hochschulbereich hat die Bedeutung der Drittmittel im Allgemeinen und der von der DFG vergebenen Drittmittel im Speziellen für die Möglichkeit zur Ausübung von Wissenschaft deutlich erhöht. Von daher stellt eine weitgehende Rechtlosigkeit der Antragsteller in den DFGFörderverfahren eine erhebliche Gefährdung der Wissenschaftsfreiheit dar. Eine solche besteht zwar nicht in den koordinierten Verfahren, wo das Verwaltungsprivatrecht gilt. Trotzdem wurden in diesen Verfahren bislang gegenüber der DFG keine Ansprüche geltend gemacht. Diese Zurückhaltung beruht wahrscheinlich darauf, dass in einem auf Konsens aufbauenden System wie dem der Wissenschaftsförderung bei Einforderung dieser Rechte beträchtliche Nachteile, insbesondere Vertrauensverluste, drohen. ${ }^{121} \mathrm{Im}$ Normalverfahren besteht sogar noch nicht einmal theoretisch die Möglichkeit, elementare Verfahrensrechte geltend zumachen. Im Hinblick auf Art. 5 GG sind Bund und Länder als fast ausschließlicher Finanzier der DFG aufgrund dessen verpflichtet, auf eine Veränderung der Vergabeverfahren hinzuwirken.

121 Streiter (o. Fußn. 19), S. 7. 